\title{
Investigation of wearing methods of a baby carrier on muscle activation during trunk flexion-extension in healthy women
}

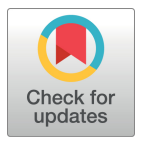

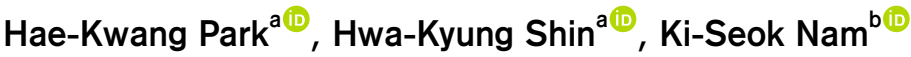 \\ a Department of Physical Therapy, Graduate School of Medical and Health Industry, Daegu Catholic University, Gyeongsan, Republic of Korea \\ ${ }^{b}$ Department of Physical Therapy, Yeungnam University College, Daegu, Republic of Korea
}

\begin{abstract}
Objective: Many caregivers often carry infants using baby carriers until they are approximately 36 months old. The purpose of this study was to compare the muscular activity of the trunk and lower leg muscles during trunk flexion-extension movements in
\end{abstract} correspondence to various wearing methods of a baby carrier blanket.

Design: Cross-sectional study.

Methods: Sixteen healthy adult women were to wear baby carrier blankets in five different ways in terms of direction and height, followed by flexion-extension of the trunk. Erector spinae (ES), rectus abdominis, rectus femoris (RF), biceps femoris (BF) muscle activities and triaxial acceleration of trunk were investigated.

Results: The front-wearing method of the baby carrier blanket increased the muscular activity of the ES muscle, and wearing the baby carrier blanket at waist height in the same direction was significantly higher than wearing it at pelvic height $(p<0.05)$. As the angle of flexion increased during trunk flexion-extension, the muscle activity of the ES, BF, and the RF increased. There was a greater increase in muscle activity of the ES and the BF during extension compared to flexion $(p<0.05)$.

Conclusions: If it is difficult to wear a baby carrier blanket due to lumbar pain, it is recommended to lower the wearing height of the baby carrier to the pelvic level so that the external load can be transferred to the lower extremity. In addition, it appears to be necessary to hold the baby and distribute the load onto the waist through proper body control when performing flexion-extension movements of the trunk. More objective and scientific research that includes various daily tasks and evaluation methods are needed.

Key Words: Biomechanical phenomena; Electromyography; Posture; Women

\section{Introduction}

In general, infants over 12 months of age who start walking have the desire to move continuously and walk outside rather than sit in a stroller, and many caregivers often carry infants using baby carriers until they are approximately 36 months old [1].

Wearing a baby carrier can increase the amount of muscle activity in the biceps femoris (BF) and vertebral muscles, and cause an imbalance in the wearer's posture by the external load of infant, and increase the risk of falling [2,3].

In particular, infants who are about 6 months old often live in the arms of their parents, and if the infants need to be held for a long time until they fall asleep or stop crying, it will be a significant burden on the musculoskeletal system of their parents [2].

When walking with an infant in front, it cannot easily bear the load and is easily biased to one side, and this imbalance is also linked to an intensive load onto the waist, causing pain and disease [4]. Holding the child in front causes a posture change similar to that of a pregnant woman, placing the center of gravity of the human body at the front of the stomach, increasing the curvature of the head and lumbar bones, increasing the level of stiffness of the torso, and increasing

Received: 5 March, 2020 Revised: 22 March, 2020 Accepted: 22 March, 2020

Corresponding author: Hwa-Kyung Shin (ORCID https://orcid.org/0000-0003-3876-0710)

Department of Physical Therapy, Graduate School of Medical and Health Industry, Daegu Catholic University, 13-13 Hayang-ro, Geumnak-ri, Hayang-eup, Gyeongsan 38430, Republic of Korea

Tel: 82-53-850-2532 Fax: 82-53-359-6772 E-mail: hkshin1@cu.ac.kr

(c) This is an Open-Access article distributed under the terms of the Creative Commons Attribution Non-Commercial License (http://creativecommons.org/licenses/ by-nc/4.0) which permits unrestricted non-commercial use, distribution, and reproduction in any medium, provided the original work is properly cited.

Copyright @ 2020 Korean Academy of Physical Therapy Rehabilitation Science 
the amount of extension of the lumbar spine, which can be a factor in increasing the amount of load onto the waist $[5,6]$. Seventy-two percent of pregnant women who gave birth experienced pain in the lumbar and hip areas, and continued to have pain in the lower back and hip areas for about 3 years after child birth [7]. The incidence of back injuries during child care is $34.1 \%$, and the incidence of back pain among mothers raising infants under 4 years of age is reported to be $48 \%$ [8,9]. A total of $87.3 \%$ of baby carrier wearers complain of low back pain, regardless of the age and weight of infants using the baby carrier [10]. For example, in the case of a Korean female, the average weight of a woman in her $20 \mathrm{~s}$ and $30 \mathrm{~s}$ is $57.3 \mathrm{~kg}$. Assuming that a child's weight ranges between 7.6 to $14.1 \mathrm{~kg}$ from 6 to 36 months and including 13 to $25 \%$ of the mother's body weight, the mother continuously carries this amount of weight on a daily basis [6]. As such, many studies have been conducted because wearing a baby carrier can place a considerable burden on the musculoskeletal system of the caregiver. According to a recent study related to this, baby carriers with shoulder straps and waist belts have the lowest burden on the body muscles, and the method of wearing the baby carriers tightly can reduce the change in shape of the spinal column $[6,11]$. Baby carriers allow the mothers to move their hands freely even when they are holding an infant or carrying a baby and therefore, a large number of women carry out household chores at the same time as raising their children, including performing the dual tasks of holding the baby while cleaning, shopping, washing dishes, etc [12]. In fact, a large number of baby carrier users often use the baby carrier to do housework, and these tasks are performed by bending the torso while holding an infant. Although many studies have been conducted to investigate how wearing a baby carrier can have an effect on putting load to the body, on gait, and on impaired spinal alignment, studies that investigate the changes in trunk flexion and extension while wearing a baby carrier are insufficient.

In addition, according to a study on the purchase and use of baby carriers, among the 345 baby carrier users, the use ratio of baby carriers with a waist support was $93.3 \%$, and of those users, $68 \%$ subjectively reported feeling fatigued. The use of baby carriers is closely related to the musculoskeletal disorders but no suggestions have been made on which height is considered to be the proper height for the lumbar support to be worn directly on the waist $[1,13]$.

Therefore, this study was designed to investigate the effects of appyling a baby carrier at different heights (waist height, pelvic height) on muscle activity during trunk flexion and extension movements. The conduction of this study was intended to provide basic data for efficient use of the muscular system and the prevention of musculoskeletal disorders.

\section{Methods}

\section{Subjects}

This study was conducted on 16 healthy adult women living in Pohang, Gyeongbuk. Those with musculoskeletal surgery experience, those with pain in the neck, back and legs when wearing a baby carrier, and those with pain during trunk flexion and extension were excluded from the study. The recruited subjects voluntarily provided their informed consent after fully understanding the purpose of the study and the overall contents of the experiment. This study was approved by the Institutional review board (IRB) of the Daegu Catholic University (IRB No. CUIRB-2019-0074). Table 1 shows the general characteristics of the study subjects.

\section{Methods}

\section{Assessment tools}

The baby carrier used in the experiment was the MAY\& MAI (domestic production), which is a commercialized product consisting of both shoulder belts, back support, neck support, and waist belt [6].

In order to imitate the average height and weight $(66 \mathrm{~cm}$, $7.6 \mathrm{~kg}$ ) of male and female infants according to various months, sandbags were applied onto the arms, legs and torso of an infant doll [6].

The Trigno wireless electromyography (EMG, Trigno Digital Lab; DELSYS Inc., Natick, MA, USA) was used to simultaneously collect EMG signals and trunk flexion and extension angles depending on how the baby carrier was worn. Trigno wireless EMG is a wireless EMG measurement device manufactured by DELSYS. Each sensor has a built-in 3-axis accelerometer $+/ 1.5 \mathrm{G},+/-4 \mathrm{G},+/-6 \mathrm{G}$,

Table 1. General characteristics of subjects

$(\mathrm{N}=16)$

\begin{tabular}{lr}
\hline \multicolumn{1}{c}{ Variable } & \multicolumn{1}{c}{ Value } \\
\hline Age $(\mathrm{y})$ & $29.2(3.89)$ \\
Height $(\mathrm{cm})$ & $162.2(4.26)$ \\
Weight $(\mathrm{kg})$ & $54.4(5.05)$ \\
\hline
\end{tabular}

Values are presented as mean (SD). 
$+/-9 \mathrm{G}$. It is a device that can simultaneously measure 16 EMG sensors and 46 accelerations. By combining the information of the acceleration and geomagnetic sensors, information on direction (inertial measurement unit [IMU] data) is provided [14]. In this study, the experiment was performed with the 3-axis accelerometer set at $2 \mathrm{~g}$, and angular values were used by analyzing the angular acceleration measured during flexion and extension.

\section{Experimental procedure}

Surface EMG was used to assess muscle activation according to the following conditions: not wearing a baby carrier (none wear, NW), worn at front-waist height (ventrolumbar, VL), worn at front-hip height (ventrosacral, VS), worn at rear-waist height (dorsolumbar, DL), and worn at rear-hip height (dorsosacral, DS).

Before attaching the electrode, body hair was removed at the site where the pad was to be attached and rubbing alcohol was used to sterilize and minimize error caused by skin resistance. The surface EMG sensor was attached to the right muscle, which is generally the dominant side, and the manual of the Surface EMG for the Non-Invasive Assessment of Muscles (SEINAM) project was referred to for electrodes to be attached to four muscles of the vertebrae (erector spinae [ES], L5 Part), rectus abdominus (RA), BF, and rectus fermoris (RF). The active electrode was applied at the height of the L5 spinous process for the ES, at the level and $2 \mathrm{~cm}$ to the side of the navel for the RA, at $1 / 2$ the distance of the line between the ischial tuberosity and the upper portion of the lateral tibial condyle for the $\mathrm{BF}$, and at $1 / 2$ the distance of the line between the anterior superior iliac spine and the upper portion of the knee for the RF.

In order to measure the angular velocity during trunk flexion and extension, two Trigno Sensors were used according to ISO 11226 Flexion Angle Measurement Standard and were attached to the spinous process of cervical 7 th $(\mathrm{C} 7)$ and the greater trochanter of the femur.

With both of the subject's feet shoulder-width apart and both hands lightly supporting the back of the infant doll at the position of his chest, the subject naturally performed trunk flexion and extension without bending their knees. In order to control the speed difference between each person during the flexion and extension tasks, the measuring instrument was performed three times during standing for $3 \mathrm{sec}-$ onds, flexion for 3 seconds, and extension for 3 seconds by notifying the counter displayed at the same time as the start.

In addition, if the body was flexed over $45^{\circ}$, the factors caused by the passive force were greater than the active force, making it difficult to accurately interpret muscle activity. A foam roller (red balance, $91.5 \mathrm{~cm}$ ) was placed on the front of the body to perform trunk flexion. Trunk flexion was controlled by repeating the movement when the hand located on the chest touched the foam roller (Figure 1) [15].

\section{Recording and processing of EMG and 3-axis accel- eration signals}

The DELSYS software EMMA was used to measure the 3-axis acceleration signal (IMU data) and the EMG signal during trunk flexion and extension motions.

The 3-axis acceleration signal (IMU data) measured during trunk flexion and extension was measured at $150 \mathrm{~Hz}$, and the EMG signal was recorded at $2,000 \mathrm{~Hz}$ and was recorded using the unit $\mu \mathrm{V}$. The measured 3-axis acceleration signal was divided into 5 sections, which were the relax standing (RS), flexion $15^{\circ} \pm 5^{\circ}(\mathrm{F} 1)$, flexion $30^{\circ} \pm 5^{\circ}(\mathrm{F} 2)$, extension $30^{\circ} \pm 5^{\circ}(\mathrm{E} 1)$, extension $15^{\circ} \pm 5^{\circ}(\mathrm{E} 2)$ analysis angles, and the EMG value corresponding thereto was saved.

The segmented EMG signal was normalized using a root mean square process to quantify, and the maximum voluntary isometric contraction (MVIC) was used. The average of three \% MVIC measurements was determined as a representative value.

\section{Statistical analysis}

SPSS for Windows, Version 12.0 (SPSS Inc., Chicago, IL, USA) was used for data analysis. The two-way repeated ANOVA was used to compare the muscle activity of each muscle according to the method of wearing the baby carrier (5) and trunk flexion and extension (5). LSD post-tests were performed to accurately interpret significant differences. The statistical significance level was set to $\alpha=0.05$.

\section{Results}

The muscle activity of each muscle according to the method of wearing the baby carrier and trunk flexion and extension were compared. There were significant differences in ES muscle activity according to the wearing method $(\mathrm{F}=$ $18.01,<0.001)$ and according to trunk flexion and extension $(\mathrm{F}=57.36,<0.001)(p<0.05)$, and there was no interaction $(\mathrm{F}=$ $0.439, p=0.971$ ) (Figure 2). According to post-hoc analysis, ES muscle activity was significantly lower with the DS method of wearing compared to $\mathrm{VL}(p<0.05)$, and there was a significant difference between all stages of torso flexion 


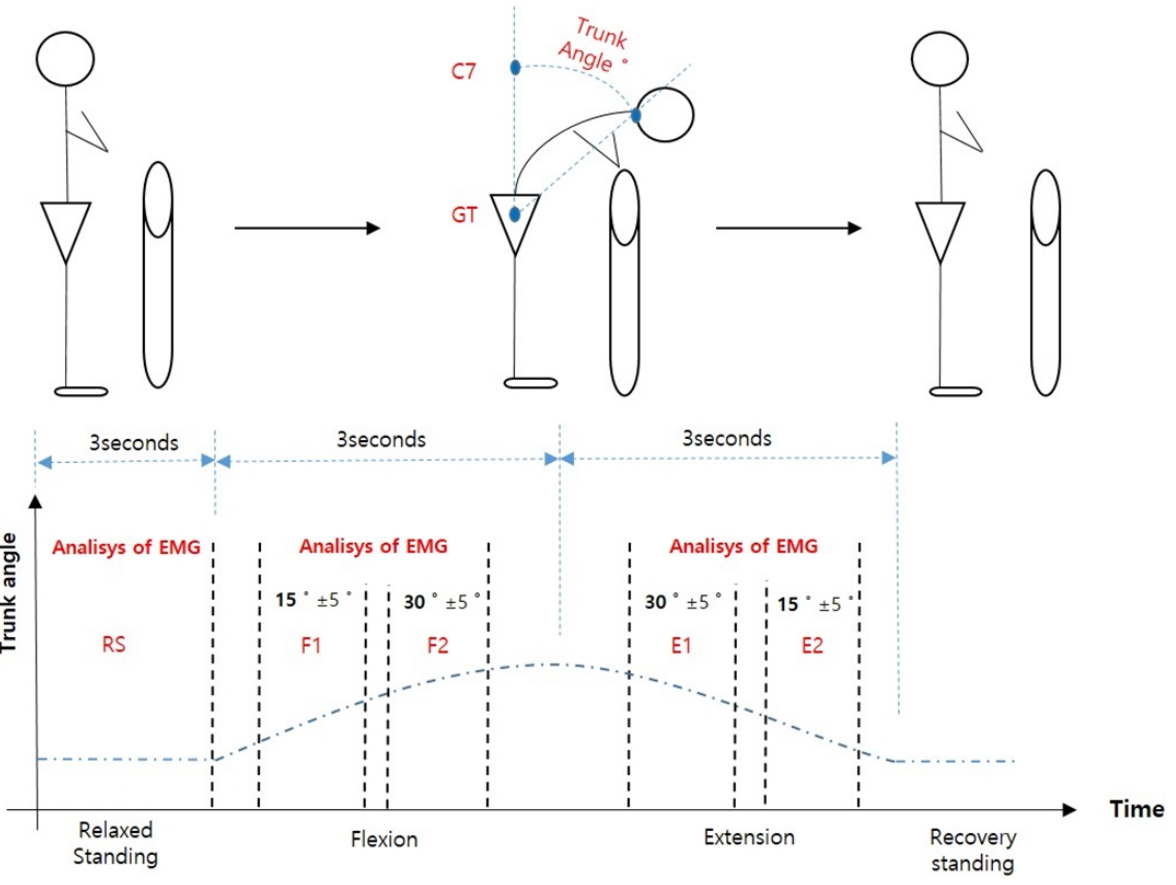

Figure 1. Analysis of electromyography during trunk flexion-extension with wearing baby carrier. $\mathrm{C}$ : : spinous process of cervical 7th, GT: great trochanter of femur, RS: relaxed standing, F1: flexion $15^{\circ} \pm 5^{\circ}, \mathrm{F} 2$ : flexion $30^{\circ} \pm 5^{\circ}$, E1: extension $30^{\circ} \pm 5^{\circ}$, E2: extension $15^{\circ} \pm 5^{\circ}$.
A

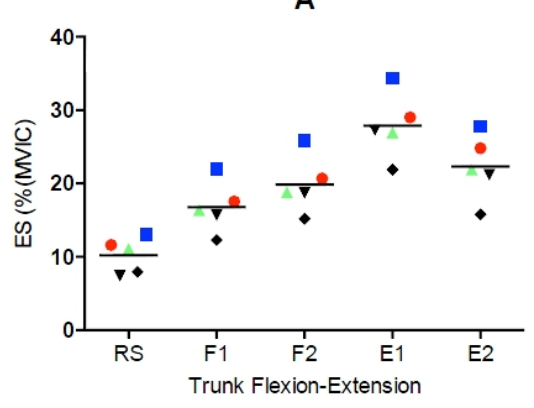

C

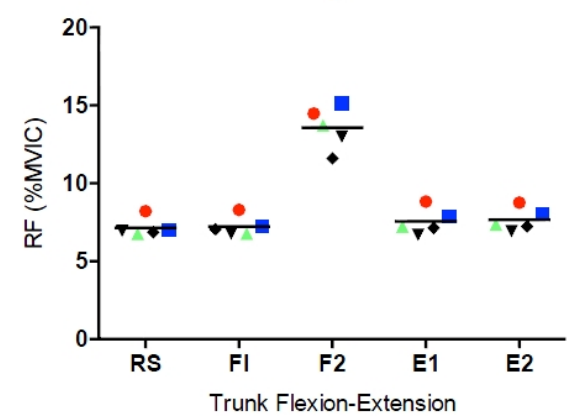

B

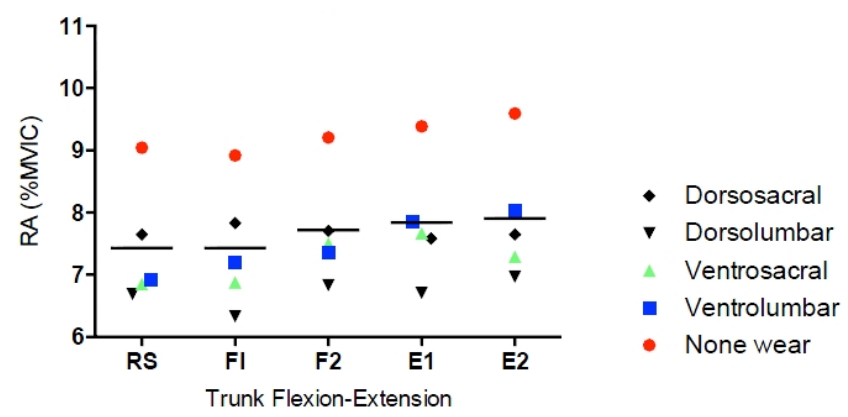

D

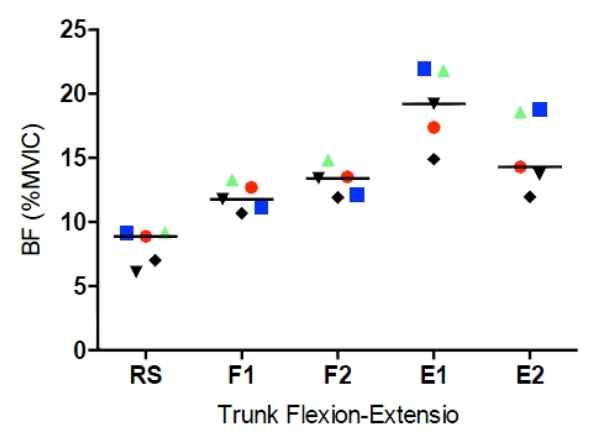

Figure 2. Muscle activation on wearing method of baby carrier during trunk flexion-extension. (A) Elector spinae (ES). (B) Rectus abdominis (RA). (C) Rectus femoris (RF). (D) Biceps femoris (BF). MVIC: maximum voluntary isometric contraction.

and extension $(p<0.05)$.

There were significant differences in muscle activity of the $\mathrm{BF}$ according to the method of wearing $(\mathrm{F}=5.674,<0.001)$ and trunk flexion and extension $(\mathrm{F}=36.075,<0.001)$, and there was no interaction $(\mathrm{F}=1.024, p=0.430)$ (Figure 2).

Post-hoc analysis showed that the DS wearing method 
was significantly lower in the $\mathrm{BF}$ than the $\mathrm{NW}$ and $\mathrm{VL}$ $(p<0.05)$, and the DL was significantly lower than the VS $(p<0.05)$. In addition, $\mathrm{F} 1$ was significantly higher than RS with the stages of trunk flexion and extension $(p<0.05)$, and E1 was significantly higher than RS, F2, and E2 $(p<0.05)$.

For the RF, there were significant differences in muscle activity according to the method of wearing ( $\mathrm{F}=18.01,<0.001)$ and stages of trunk flexion and extension $(\mathrm{F}=57.36,<0.001)$, and there was no interaction ( $\mathrm{F}=0.439, p=0.971)$ (Figure 2). According to post-hoc analysis, the RF muscle activity was significantly lower with the DS method compared to the NW $(p<0.05)$, and the VL and DL methods were significantly lower compared to the NW method $(p<0.05)$. According to the stages of trunk flexion and extension, the $\mathrm{F} 2$ stage was significantly higher than the other stages $(p<0.05)$.

On the other hand, there were no significant differences in RA muscle activity according to the method of wearing $(\mathrm{F}=3.665, p=0.06)(p<0.05)$ and stages of trunk flexion and extension ( $\mathrm{F}=0.260, p=0.935)(p>0.05)$, there was no interaction $(\mathrm{F}=0.047, p=1.000)$ (Figure 2).

\section{Discussion}

Pawlowski and Grabarczyk [16] reported that the lower the center of gravity compared to the kidneys, the better the stability of carrying during pregnancy and infants. In this study, the height of the waist support of the baby carrier, which was worn by 16 healthy adult women, was changed, and the method of wearing the waist support at the front and back of the pelvis was added in addition to the general method of wearing it on the front and back of the hips. Muscle activity of the erector spinae, RA, RF, and BF muscles were compared according to the method of wearing a baby carrier and during various stages of trunk flexion and extension. Muscle activity was analyzed according to the five various methods of wearing a baby carrier as well as the five stages of trunk flexion and extension and results showed that there were statistically significant differences between the ES, $\mathrm{RF}$, and the $\mathrm{BF}$ muscle activities according to the wearing method and according to trunk flexion and extension stages.

The muscle activity of the ES muscles according to each wearing method of the baby carrier showed greater muscle activity when worn anteriorly compared to posteriorly. This may have occurred in order to move the center of the external load located in front of the waist close to the body center line, and the lumbar muscles are required to generate an internal force against the external load [17].
It was found that the external load located in front of the waist required greater activity of the ES muscles [18]. In addition, although worn in the same direction, the muscle activity was different according to the differing heights of the application of the baby carrier. It was found that wearing at waist height showed greater muscle activity than wearing at pelvis height, and the lower the height of the lumbar support, the lower the muscle activity of the ES muscles.

This is a result of the central height of the hip and external loads where movement occurs, and the center of head and arms (HAT), and body is located directly under the xiphoid process, and the action of leaning forward and standing up again increases the distance from the center of hip joint to the center of the HAT, requiring more muscle strength in the hip and spine muscles $[19,20]$.

That is, it is predicted that the baby carrier worn at waist height has a higher center of gravity of the HAT than the baby carrier worn on the pelvis, and the external load is more weighted on the weight of the HAT during trunk flexion and extension.

Of the anterior wearing methods, the pelvic height wearing method showed lower muscle activity of the ES muscles than the waist height wearing method, whereas the lumbar support worn at the pelvic height showed the greatest muscle activity in the BF muscle. The waist support worn at the height of the pelvis acts as an external load to the anterior load of the pelvis, which is accompanied by trunk flexion to further increase the anterior inclination. The biceps muscle is considered to be the most active in controlling and supporting the anterior tilt of the pelvis [20].

The application method that exhibited the lowest muscle activity of the ES muscle was the posterior pelvic height method.

These results suggest that the external load located on the back acts as the contraction of the ES muscles during trunk flexion and extension, and thus has a mechanical benefit. During trunk flexion and extension, the ES and BF muscle activity increased with flexion, and showed greater muscle activity during trunke extension than flexion [21,22]. As a result, in regards to muscle activity while performing trunk flexion from an upright standing position, the greater the flexion, the greater the moment arm, and eccentric contraction activity is increased in order to control the speed of trunk flexion [23].

Although there was no significant difference in muscle activity of the RA according to the wearing method, all subjects wearing a baby carrier showed lower muscle activity 
than those not wearing a baby carrier. This is different from the results of previous studies that have shown that activity of the RA increased when the baby carrier was worn backwards. As a result, it is thought that the abdominal pressure passively increases due to wearing a waist belt [24].

The muscle activity of the RF according to the wearing method showed a significant difference only with the nonwearing group, and there was no significant difference between the groups wearing the baby carrier, showing similar muscle activity. However, there was higher muscle activity during the F2 stage of trunk flexion and extension, and it can be considered that and as soon as the trunk flexion exceeded a certain point, the load was transferred to the RF muscle [25].

As a result of this study, the anterior application of a baby carrier required greater muscle activity of the ES muscles than the posterior application, and wearing at the anterior hip level compared to the anterior pelvic level increased the muscle activity of the BF and decreased muscle activity of the ES. Large forces created by the ES muscles can damage the muscles, intervertebral discs, bony joints of the spine [26]. Therefore, it was found that it is necessary to move the load to the leg muscles by lowering the wearing height in order to reduce fatigue and pain of the ES muscles when wearing the baby carrier anteriorly. In addition, the ES muscles appear to require greater muscle activity during the trunk extension compared to flexion, and it seems necessary to distribute the load to be applied to the passive structures of the vertebral muscles and lower back through the adjusted leg movement during the trunk flexion and extension [27].

Caregivers who have to wear a baby carrier for a long period of time during their daily lives must properly exercise in order to prevent muscle atrophy of the lumbar ES muscles and stabilize the lower back and pelvis to prevent back pain. It is necessary to avoid continuously wearing the baby carrier in one fixed posture and choose an efficient wearing method for daily life function and to prevent discomfort in the lower back [28-30].

In this study, although the weight of the infant doll was fixed, the weight of the subjects could not be controlled. Also, the subjects were not distinguished based on those who've had experience with using a baby carrier versus those with no experience, and results could not include variables such as an increase in the infant's weight and movements created by the infant since this study used infant dolls. In addition, it is difficult to explain all of the dynamics of the body using only with muscle activity that occurs during trunk flexion and extension. Therefore, in the future, it will be necessary to include more closely related tasks in everyday life and to conduct objective research on actual infants and caregivers.

\section{Conflict of Interest}

The authors declared no potential conflicts of interest with respect to the authorship and/or publication of this article.

\section{References}

1. Lee HR, Lee YJ. A survey on the purchase and use of baby carriers. Korean J Hum Ecol 2017;26:157-70.

2. Yuk GC, Park RJ, Lee HY, Lee MH, Lee JH, Kuk JS, et al. The effects of baby carrier and sling in muscle activation of trunk, low extremity and foot pressure. J Korean Soc Phys Med 2010;5: 223-31.

3. Frisbee SJ, Hennes H. Adult-worn child carriers: a potential risk for injury. Inj Prev 2000;6:56-8.

4. Wall-Scheffler CM, Geiger K, Steudel-Numbers KL. Infant carrying: the role of increased locomotory costs in early tool development. Am J Phys Anthropol 2007;133:841-6.

5. Singh $E$. The effects of various methods of infant carrying on the human body and locomotion. [Bachelor thesis]. Delaware: University of Delaware; 2009.

6. Lee HR, Hong KH. Alteration of the spine shape depending on the wearing method and type of baby carrier. Korean J Hum Ecol 2017;26:435-44.

7. Norén L, Ostgaard S, Johansson G, Ostgaard HC. Lumbar back and posterior pelvic pain during pregnancy: a 3-year follow-up. Eur Spine J 2002;11:267-71.

8. Padua L, Caliandro P, Aprile I, Pazzaglia C, Padua R, Calistri A, et al. Back pain in pregnancy: 1-year follow-up of untreated cases. Eur Spine J 2005;14:151-4.

9. Sanders MJ, Morse T. The ergonomics of caring for children: an exploratory study. Am J Occup Ther 2005;59:285-95.

10. Ojukwu CP, Fab-Agbo C, Ikele CN, Onuchukwu CL, Anekwu EM. Infant carrying-related low back pain: prevalence and correlates among nursing mothers in Enugu, Nigeria. Int J Med Biomed Res 2017;6:125-35.

11. Yu YJ, Lee KK, Lee JH, Kim SB. Effects of transporting a baby with varied baby carriers on the posture of mother during gait. Asian J Kinesiol 2018;20:45-51.

12. Anderson AM, Meador KA, McClure LR, Makrozahopoulos D, Brooks DJ, Mirka GA. A biomechanical analysis of anterior load carriage. Ergonomics 2007;50:2104-17.

13. Lee HR, Hong KH. Evaluation of muscle fatigue and subjective fatigue depending on the hip seat and waist support band of baby carrier. Fash Text Res J 2017;19:504-14.

14. Saber-Sheikh K, Bryant EC, Glazzard C, Hamel A, Lee RY. Feasibility of using inertial sensors to assess human movement. Man Ther 2010;15:122-5.

15. McGill SM, Norman RW. Partitioning of the L4-L5 dynamic 
moment into disc, ligamentous, and muscular components during lifting. Spine (Phila Pa 1976) 1986;11:666-78.

16. Pawłowski B, Grabarczyk M. Center of body mass and the evolution of female body shape. Am J Hum Biol 2003;15:144-50.

17. Cook TM, Neumann DA. The effects of load placement on the EMG activity of the low back muscles during load carrying by men and women. Ergonomics 1987;30:1413-23.

18. Lee SY, Chang JS, Lee MH. The effects of plantar foot pressure and EMG activation of neck, lumbar and low limbs by using carrier during walking. Korean J Sport Biomech 2009;19:237-44.

19. Elftman H. Knee action and locomotion. Bull Hosp Joint Dis 1955;16:103-10

20. Marshall PW, Mannion J, Murphy BA. The eccentric, concentric strength relationship of the hamstring muscles in chronic low back pain. J Electromyogr Kinesiol 2010;20:39-45.

21. Andersson EA, Oddsson LI, Grundström H, Nilsson J, Thorstensson A. EMG activities of the quadratus lumborum and erector spinae muscles during flexion-relaxation and other motor tasks. Clin Biomech (Bristol, Avon) 1996;11:392-400.

22. McGorry RW, Hsiang SM, Fathallah FA, Clancy EA. Timing of activation of the erector spinae and hamstrings during a trunk flexion and extension task. Spine (Phila Pa 1976) 2001;26:41825.

23. Floyd WF, Silver PH. The function of the erectores spinae muscles in certain movements and postures in man. J Physiol 1995;
129:184-203

24. Chang JS, Lee SY, Lee MH, Kim JH, Kim CY. The effects of EMG activation of neck, lumbar and low limb by using baby carrier with arms during walking. J Korean Soc Phys Med 2010;5: 323-30.

25. Trafimow JH, Schipplein OD, Novak GJ, Andersson GB. The effects of quadriceps fatigue on the technique of lifting. Spine (Phila Pa 1976) 1993;18:364-7.

26. Neumann DA, Rowan EE. Kinesiology of the musculoskeletal system : foundations for physical rehabilitation. St. Louis: Mosby; 2002. P. $370-8$.

27. Kang DH, Lee WH, Lim S, Kim YY, An SW, Kwon CG, et al. The effect of hip joint exercise using an elastic band on dynamic balance, agility and flexibility in healthy subjects: a randomized controlled trial. Phys Ther Rehabil Sci 2016;5:198-204.

28. Shin DC, Song CH. Relationship of trunk muscle atrophy and provocation position in patients with chronic low back pain. Phys Ther Rehabil Sci 2012;1:28-32.

29. Chai W, Lee SH, Park YH. The effect of co-contraction exercises of abdominal bracing combined with ankle dorsiflexion on abdominal muscle thickness and strength in patients with chronic low back pain. Phys Ther Rehabil Sci 2014;3:93-100.

30. Lee J, Jeong K, Lee H, Shin J, Choi J, Kang S, et al. Comparison of three different surface plank exercises on core muscle activity. Phys Ther Rehabil Sci 2016;5:29-33. 